\title{
ELECTRIC GENERATOR IN THE RECUPERATION SYSTEM OF THE ENERGY FROM MECHANICAL OSCILLATIONS IN VEHICLES
}

\begin{abstract}
The paper deals with the system of mechanical energy recuperation of vehicles in the useful electric energy accumulated in the rechargeable battery. This system creates an additional power supply on board of the vehicle and, based on the principle of energy conservation, increases the efficiency of the use of the fuel of the primary engine. One of the main elements of such system is an electric generator, which transforms the mechanical energy of the oscillations of the vehicle's chassis into electric energy. The problem of choosing and optimizing the design and parameters of the generator is considered in the paper. Given the peculiarities of the functioning of the vehicle, the most appropriate type of generator in such system is a synchronous generator with permanent magnet, which has significant structural differences from conventional synchronous machines with permanent magnets. The criterion for optimizing the generator is the largest value of the effective value of the EMF, which is induced in the stator winding. On the basis of simulation results, based on the field mathematical model, a set of values of generalized coefficients that characterize the optimal generator geometry is obtained. References 4, table 1, figures 7.

Key words: electric energy recuperation system, synchronous generator, permanent magnets, mathematical model.
\end{abstract}

В статье рассматривается система рекуперации энергии механических колебаний транспортных средств в электроэнергию, которая накапливается в аккумуляторной батарее. Одним из основных элементов такой системы является электрогенератор, который преобразует механическую энергию колебаний иисси транспортного средства в электроэнергию. Также рассмотрена проблема выбора и оптимизации конструкции и параметров генератора. Учитывая особенности функциониования транспортного средства наиболее целесообразным типом генератора в такой системе является синхронный генератор с постоянными магнитами, который имеет существенные конструктивные отличия от традиционных синхронных машин с постоянными магнитами. Критерием оптимизации генератора является наибольшая величина действующего значения ЭДС, индуцированной в обмотке статора. По результатам моделирования на основе полевой математической модели получена совокупность значений обобщенных коэффициентов, характеризующих оптимальную геометрию генератора. Библ. 4, табл. 1, рис. 7.

Ключевые слова: система рекуперации электроэнергии, синхронный генератор, постоянные магниты, математическая модель.

Introduction. Requirements for expanding the functionality of modern vehicles of general and special purpose cause the use on board of the vehicles of new devices and systems, operation of which requires additional power supply sources. Since all the energy received by the vehicle for its operation comes from the use of primary energy sources (usually the fuel of an internal combustion engine), additional power sources can only be obtained on the basis of energy saving principles - by detecting unnecessary energy losses in the vehicle and developing systems for the conversion of a part of these losses in useful power storage of battery. One of these is the system of electromechanical conversion of the energy of mechanical oscillations (SCEMO) of the vehicle's chassis, which arise during the movement of the vehicle. The development and research of such systems is already being carried out by a number of foreign companies - Bose, Levant Power Corp, Audi [1-3]. For example, the power of the eROT system for an Audi car is up to $613 \mathrm{~W}$ in poor coverage, and $100-150 \mathrm{~W}$ on conventional roads. In Ukraine, these developments are just beginning, and their use may be the most expedient and effective for heavy duty vehicles.

The goal of the paper is obtaining by the results of mathematical modeling of optimal parameters of the synchronous generator, which is part of the system of electromechanical transformation of the energy of mechanical vibrations of the vehicle chassis.

General analysis. At a uniform motion of the vehicle on an ideally smooth surface (road), there is a balance of the forces of gravity of the subspring mass
(SSM) of the vehicle and the reaction of the elastic element of the suspension. In this case, the SSM does not make vertical vibrations, and the engine power is spent only to overcome the force of friction of the wheels on the surface. In the presence of steps down of the depth $h$ on the road, the wheels of the vehicle are rapidly lowered down and, under the influence of the imbalance of forces, the SSM drops down, reducing its potential energy by the value

$$
\Delta W=m g h,
$$

where $m$ is the mass of the SSM, $g$ is the acceleration of gravity.

After the occurrence of damped oscillations, as a result of which the indicated energy dissipates in springs of suspension, the mode of uniform movement of the vehicle is restored. In the presence of steps up on the road, the potential energy of the SSM is increased due to the energy of the drive motor. In the process of oscillations, there is a mutual movement of the nonsubspring mass (NSSM) and subspring mass of the vehicle in the vertical plane, which allows the creation of systems of electromechanical conversion of the energy of mechanical oscillations into useful electric energy. In the presence of a number of steps (unevenness, pits) on the road, this process is cyclically repeated. In real conditions, the frequency of pits on the road and their depth are random. However, to estimate the additional energy losses caused by vertical fluctuations of the vehicle when driving on a rough road, an equivalent

(C) Yu.M. Vaskovskyi, M.V. Poda, I.V. Koshikar 
frequency of the repetition of the oscillation process $f$ can be introduced at the same depth $h$ of pits. Frequency of natural oscillations of the SSM of the vehicle is usually adjusted to a value of $1 \ldots 2 \mathrm{~Hz}$, and they, due to powerful shock absorbers, damp in a few oscillations.

We consider examples of heavy vehicles: 1) a truck $\mathrm{KrAZ}-253 \mathrm{~B}$, its curb weight (CW) is 11.5 tons; 2) an armored personnel carrier BTR-4 Bucephalus - CW is 21.9 tons. Assuming that the ratio of NSSM and SSM is $1: 15$, which is typical for any vehicle, the values of SSM are respectively 10.7 and 20.4 tons. At one oscillation of such a mass at a depth $h=0.05 \mathrm{~m}$, the change in the potential energy of the SSM will be $\Delta W=5.24$ and $10 \mathrm{~kJ}$ respectively. If, when moving vehicle in rough terrain, such oscillations are constantly repeated, for example, with frequency $f=0.2 \mathrm{~Hz}$ (period of oscillation is $5 \mathrm{~s}$ ), then the losses of power in shock absorbers of the vehicle are:

$$
\Delta P=\Delta W \cdot f
$$

or respectively $\Delta P=1.05$ and $2 \mathrm{~kW}$. The obtained estimation testifies to considerable losses of power of the engine of the vehicle in shock absorbers, which makes it expedient for its transformation into electric energy, its accumulation in the battery and further use in consumer systems of the vehicle.

For an integral estimation of the power of mechanical oscillations, taking into account the various factors influencing its value, one can introduce the coefficient of power of mechanical oscillations

$$
k_{e}=m h f,(\mathrm{t} \cdot \mathrm{m} / \mathrm{s}) \text {. }
$$

So, for the examples given, at $\Delta P=1.05 \mathrm{~kW}$, we have $k_{e}=0.107$, and at $\Delta P=2 \mathrm{~kW}$ the coefficient is $k_{e}=0.204$. It is obvious that the same values of $\Delta P$ can be obtained with other values of mass, depth of the step and frequency of repetition of oscillations. For example, $\Delta P=1.05 \mathrm{~kW}$ can be obtained at $m=5.36 \mathrm{t}, h=0.05 \mathrm{~m}$ and $f=0.4 \mathrm{~Hz}$ or at $m=8 \mathrm{t}, h=0.06 \mathrm{~m}$ and $f=0.23 \mathrm{~Hz}$. Taking into account the expressions (1) and (2) we have a simple universal formula for estimating the power losses during the movement of a vehicle on an uneven road:

$$
\Delta P=g k_{e} .
$$

The reciprocal movement of the SSM and NSSM during the drive in difficult road conditions provides a fundamental opportunity for the creation of SCEMO of the vehicle.

Structural flowchart of the SCEMO is presented in Fig. 1.

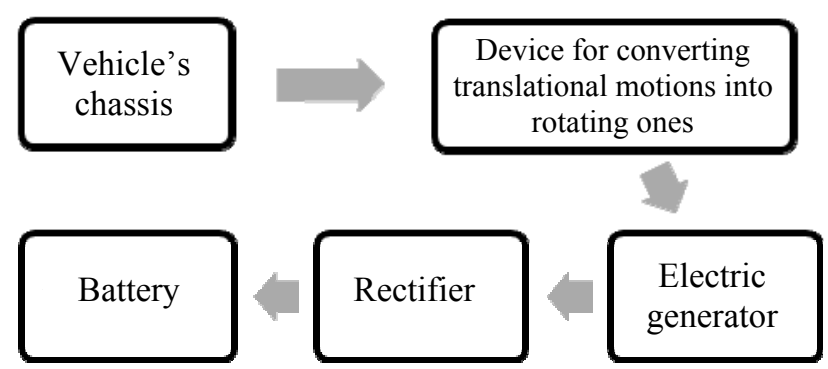

Fig. 1. Structural flowchart of the SCEMO

The operation of SCEMO is as follows. The mutual reciprocating translational displacements of the SSM and
NSSM through a mechanical transforming device are converted into a unidirectional rotational motion of the rotor of the electric generator, in which the conversion of mechanical energy into electrical energy is carried out. The alternating voltage at the output of the generator rectifies in the rectifier and charges the battery. Thus, in SCEMO there is a transformation of energy parameters in the following sequence: «mechanical energy of the reciprocating translational motion - mechanical energy of the rotating motion - electrical energy of alternating current of the electric generator - DC electric power of battery».

The electric generator creates a braking electromagnetic torque that counteracts the forced oscillation of the vehicle chassis. Thus, SCEMO carries out two useful functions: a) converts a part of the energy of mechanical vibrations of the vehicle into the useful electric power of battery; b) promotes dumping of the chassis oscillations, that is, partially serves as a shock absorber. It should be noted that the second function in heavy vehicles is not the main, because SCEMO in heavy vehicles can not replace the traditional shock absorbers, or can only to a small extent supplement them. Thus, the main function of SCEMO is the creation on board of the vehicle of an additional power source.

Modeling and investigations of the electric generator of SCEMO. An important element, which largely determines the efficiency of energy recuperation in SCEMO, is an electric generator. The analysis shows that, taking into account the features of the SCEMO operation, the optimal generator type is a three-phase AC synchronous generator with permanent magnets on the rotor (SGPM).

The use of permanent magnets (PM) as sources of magnetic flux of excitation is the optimal technical solution. In this case, the generator does not have a long stable mode of operation - it always operates in transient modes with variable speed of rotation of the shaft. The mechanical torque leading the generator in rotation has a random impulse character due to the road conditions of the motion of the vehicle. The main issues of the design of an electric generator are: a) optimization of the design of the generator by the chosen criterion, taking into account the probabilistic variables of the characteristics of the traffic of the vehicle; b) estimation of the possible share of the energy of mechanical vibrations of the vehicle, which can be converted into electric energy, taking into account the allowable overall characteristics of SCEMO.

Taking into account the real conditions of the functioning of the SGPM in the SCEMO structure, its design and parameters have a number of significant differences from the SGPM of the traditional execution. Namely:

1. An analysis of the appropriate construction of the SGPM on the vehicle chassis shows that the general configuration of the generator should be «long» - the ratio of the length of the stator core to its outer diameter lies in the range of values $l_{\delta} / D_{j}=2.5 \ldots 3.5$.

2 . The average speed of rotation of the rotor, which the generator can receive in the SCEMO, is relatively small: 
$n_{2}=200 \ldots 400 \mathrm{rpm}$. Therefore, in order to obtain an acceptable value of the EMF of the SGPM stator winding, which is proportional to the velocity of the change in the time of magnetic flux linage (frequency), the generator must have a sufficiently large number of poles - the number of pairs of poles is $p=4 \ldots 5$.

3. Taking into account the possibilities of placing a multipolar three-phase winding of the stator in the core of a small diameter, the number of coils per pole and phase of the stator winding is equal to $q=1$, and the number of grooves in the stator core is $Z_{1}=24 \ldots 30$. Taking into account these data, stator design is performed according to the general methods of designing $\mathrm{AC}$ electric machines.

4. In order to ensure a high level of reliability of SGPM it is expedient to use a rotor with PM, which have a radial direction of the magnetization vector and are fixed on the surface of the ferromagnetic yoke of the rotor. Fig. 2, as an example, shows the cross-section of the active zone of the variant of the SGPM.

5. Important is the correct choice of PM parameters. It is necessary to justify the criteria by which this choice should be fulfilled. Unlike the traditional SGPM, for a generator operating in SCEMO there is no requirement for a sinus distribution of magnetic flux density in the air gap along the pole divider. The EMF and the stator currents do not necessarily have to change in time under the sinusoidal law, since the load of the SGPM in the SCEMO is a rectifier and further battery (Fig. 1). The criterion for choosing PM parameters is the maximum value of the effective value of the EMF of the stator winding when performing other equal conditions.

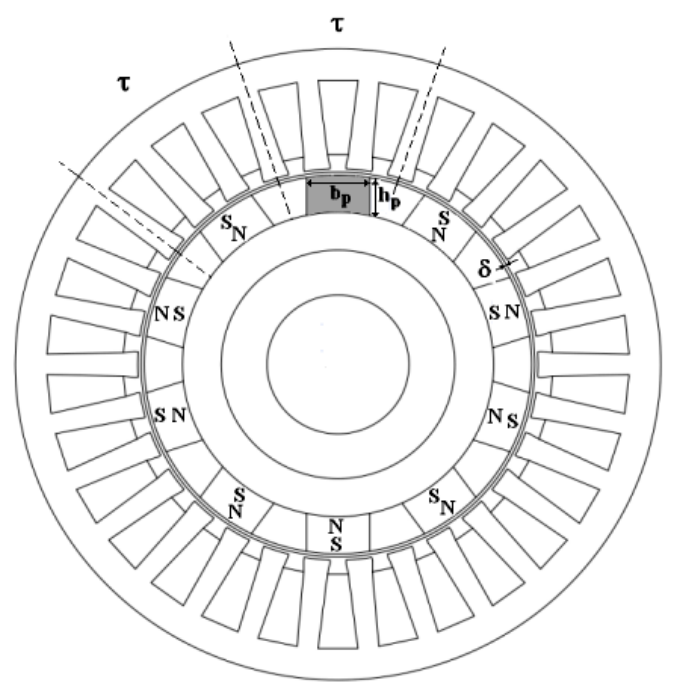

Fig. 2. Cross-section of the active zone of the SGPM

Generalized geometric parameters that characterize PM in the design of SGPM are:

- magnet shape factor - magnet width ratio to its height $k_{p}=b_{p} / h_{p}$. Typically, considering the requirements for the simplicity and performance of the rotor, PM is performed in the as a prism with a rectangular cross-sectional shape;

- coefficient of pole overlap - ratio of the width of the rotor magnet to the pole section of the rotor $\alpha=b_{p} / \tau$;
- coefficient of height of a magnet that characterizes the ratio of the height of the PM to the radius of the rotor $k_{R}=h_{p} / R_{2}$;

- coefficient of the relative value of air gap - the ratio of the height of the air gap between the stator and the rotor to the pole division of the rotor $k_{\delta}=\delta / \tau$.

The variation of the indicated coefficients in optimizing the parameters of a magnet should occur under the condition of constant variation in each variant of the cross section (volume) of the PM $S_{p}=b_{p} \cdot h_{p}=$ const, since an increase in the volume of PM, and hence its magnetic flux, in itself increases the EMF of the stator and therefore does not allow to determine the optimal geometry of SGPM.

The indicated coefficients characterize not only the geometry of the PM itself, but also its relation with the geometry of other elements of the active zone of SGPM. Thus, the optimization problem is reduced to finding the extremum of the objective function - the maximum of the effective value of the EMF of the stator winding when the specified coefficients are varied, taking into account the constraints on their values, which arise from the possibilities of constructive execution of the generator. This optimization is performed by mathematical modeling.

The mathematical model of SGPM. We consider a field mathematical model of the SGPM with a moving rotor, which allows to analyze in time electromagnetic processes that arise during rotation of a rotor [4]. Equations for temporal functions are written as follows:

$$
\begin{gathered}
\nabla \times\left(\frac{1}{\mu}\left(\nabla \times \boldsymbol{A}-\boldsymbol{B}_{\boldsymbol{r}}\right)\right)+\gamma \frac{\partial \boldsymbol{A}}{\partial t}-\gamma \boldsymbol{v} \times(\nabla \times \boldsymbol{A})=\boldsymbol{J}, \\
E_{j}(t)=\frac{2 n a l_{\delta}}{S_{P}} \int_{S_{i}} E_{n}(t) d s,
\end{gathered}
$$

where: $\boldsymbol{A}$ is the magnetic vector potential; $\boldsymbol{B}_{\boldsymbol{r}}$ is the vector of residual magnetic flux density of the PM; $\mu, \gamma$ are the magnetic permeability and electrical conductivity of the material (given for each sub-region of the calculation area according to their characteristics - PM, air gap, ferromagnetic steel, etc.); $\boldsymbol{v}$ is the vector of speed of movement of sections of the rotor relative to the system of coordinates of the stator; $J=I_{j} n a / S_{P}$ is the current density in the stator winding, which is determined by the given current values, the number of successively connected conductors in the groove of the stator $n$ and the part of the cross-section of the stator groove $S_{P}$, which falls on one parallel branch.

The $\boldsymbol{B}_{\boldsymbol{r}}$ vector should reflect the chosen direction of magnetization of the PM. The speed vector is determined by the given angular speed of the rotor $\omega_{R}$ and the radius vector of the current rotor point $\boldsymbol{v}=\omega_{R} r$.

The equation (5) is supplemented by the corresponding boundary conditions. Usually this is the homogeneous boundary conditions of the first kind on the external boundary of the calculated area - the yoke of the stator core.

The instantaneous EMF of the stator phase as a function of time is determined by formula (6), where 
integration is performed on the total area of the crosssection of phase conductors. The normal component of the electric field strength vector $E_{n}(t)$ is integrated - a component of the strength vector, which is directed perpendicular to the cross-section of the conductors of the winding. The electric field strength, provided that the electric scalar potential $\varphi=0$, is calculated as

$\boldsymbol{E}(t)=-\partial \boldsymbol{A} / \partial t$. The current value of the EMF is calculated on the period $T$ of the change of the function $E_{j}(t)$

$$
E_{r m s}=\sqrt{\frac{1}{T} \int_{0}^{T} E_{j}^{2}(t) d t} .
$$

At rotor rotation, the configuration of the calculated area is changed due to changes in the relative position of the stator and elements of the design of the rotor, that is, changes in the coordinates of all points of the rotor. At rotation of a rotor counterclockwise with constant speed, the growth of coordinates of rotor points $\Delta x, \Delta y$ in one time step $\Delta t$ is determined:

$$
\left\{\begin{array}{l}
\Delta x=\cos \left[\omega_{R} \cdot(t+\Delta t) \cdot X-\sin \left[\omega_{R} \cdot(t+\Delta t)\right] \cdot Y-X\right] \\
\Delta y=\sin \left[\omega_{R} \cdot(t+\Delta t) \cdot X+\cos \left[\omega_{R} \cdot(t+\Delta t)\right] \cdot Y-Y\right]
\end{array},\right.
$$

where $X, Y$ are the current coordinates of rotor points.

Results of simulation and optimization of SGPM. SGPM is considered, which has the following data: number of stator phases $m=3$; number of pairs of poles $p=5$; active length $l_{\delta}=0.26 \mathrm{~m}$; outer diameter of the yoke of the stator $D=0.1 \mathrm{~m}$; inner diameter of the yoke of the rotor, $D_{0}=0.022 \mathrm{~m}$; nominal power $P_{n}=400 \mathrm{~W}$; rated (active) phase voltage of the stator winding $U_{1}=16 \mathrm{~V}$; rated rotor rotation speed $n_{\text {nom }}=200 \mathrm{rpm}$ The permanent magnet has the following characteristics: $B_{r}=1.18 \mathrm{~T}$, $\mu_{r}=1.065$.

Fig. 3 shows the time dependencies of the stator EMF for different values of the pole overlay coefficient: $1-$ for $\alpha=0.69 ; 2-\alpha=0.58 ; 3-\alpha=0.49 ; 4-\alpha=0.36$. From the above dependencies it is seen that the growth of $\alpha$ leads to an increase in the width of the curves, and hence to an increase in the effective value of the EMF at its practically unchanged amplitude. That is, the width of the PM substantially affects the value of $E$.

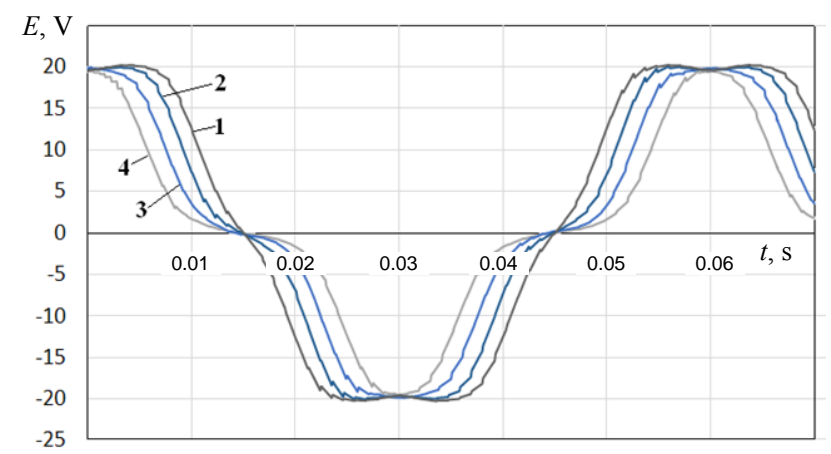

Fig. 3. Temporal dependencies of EMF

Calculations are made in idle mode of the generator. Fig. 4, 5 show the dependencies of the amplitude (dotted line) and the active value (solid line) of the EMF of the stator winding on, respectively, the pole overlay $\alpha$ and the shape coefficient of the magnet $k_{p}$ on the condition that the volume of the PM is unchanged.

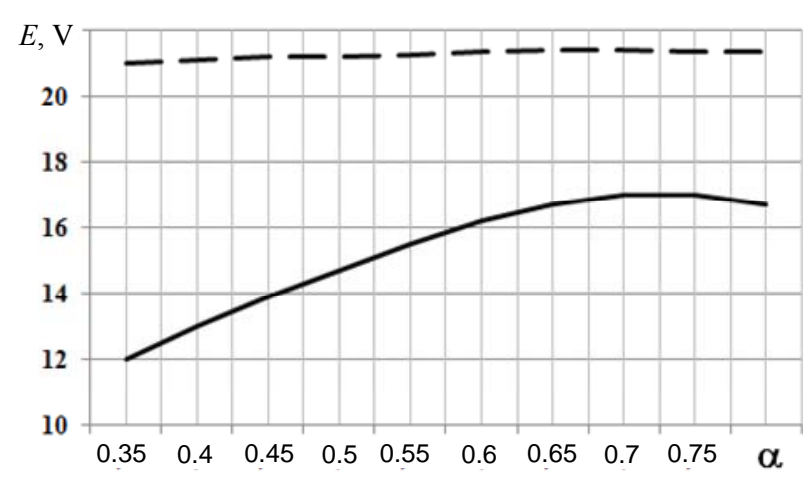

Fig. 4. EMF dependencies on the coefficient $\alpha$

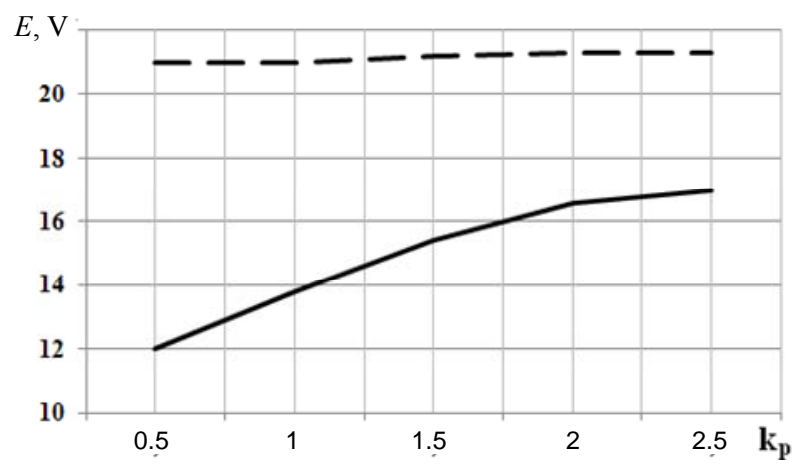

Fig. 5. EMF dependencies on the coefficient $k_{p}$

The presented dependencies show that with practically unchanged amplitude of the EMF the use of wider and not high PM leads to an increase in the effective value of EMF.

The excessive increase in the width of the PM is not feasible, since it is limited by an increase in the magnetic scattering fluxes between the PMs that are located near, as well as by constructive factors. The optimal values of the coefficients are: $k_{p}=2.5 \ldots 3$ and $\alpha=0.75 \ldots 0.8$.

This conclusion is confirmed by the calculated dependences of the EMF on the coefficient of magnet height $k_{R}=h_{p} / R_{2}$, shown in Fig. 6. Calculations are made with constant magnet width $b_{p}=6 \mathrm{~mm}$ and variation of the height of the magnet from 3 to $8 \mathrm{~mm}$. With variations, the volume of magnets increases by 2.67 times. The given data testify that at $k_{R}>0.2$ the growth of EMF does not occur, that is, the performance of too high magnets is inappropriate.

This is due to the fact that the part of the PM, located near the yoke of the rotor, practically does not «take part» in the creation of the magnetic flux of the mutual induction of the rotor with the winding of the stator, and only creates fluxes of scattering of PM. Therefore, the values of the magnitude of the coefficient of magnet height lie in the range of values $k_{R}=0.1 \ldots 0.13$.

The size of the air gap between the stator and the rotor affects the EMF of the winding of the stator. Fig. 7 shows the temporal dependencies of the EMF of the winding of the stator for the values: $\delta=0.5 \mathrm{~mm}$ (indicated by 1 ); $0.75 \mathrm{~mm} \mathrm{(2)}$ and $1 \mathrm{~mm} \mathrm{(3),} \mathrm{which} \mathrm{correspond} \mathrm{to} \mathrm{the} \mathrm{value}$ of the coefficient of the relative air gap value $k_{\delta}=\delta / \tau=$ $=0.053 ; 0.04$ and 0.0265 . 


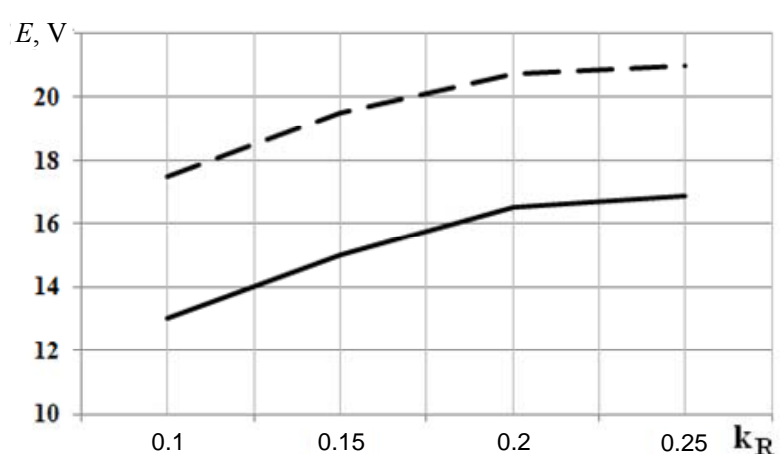

Fig. 6. EMF dependencies on the coefficient $k_{\mathrm{R}}$

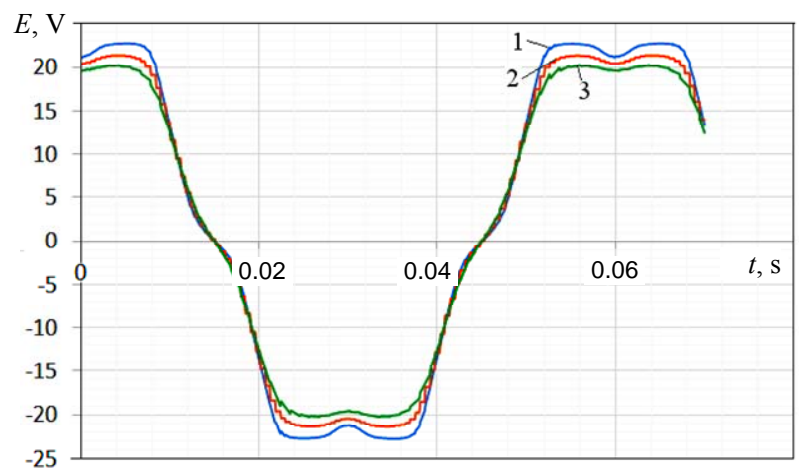

Fig. 7. Temporal EMF dependencies on the coefficient $k_{\delta}$

Calculations are carried out at a fixed value of the width of the PM. From the data shown, it can be seen that even with a double magnification of $\delta$ within the limits which can be due to constructive factors, the amount of EMF decreases by only $13.7 \%$.

Similar numerical studies were carried out for other standard sizes of SGPM, which resulted in the presented in the Table optimal values of generalized geometric coefficients, which should be used in the design of SGPM, which are intended for operation in SCEMO.

Table

SGPM optimal geometrical coefficients

\begin{tabular}{|l|c|}
\hline \multicolumn{1}{|c|}{ Coefficient } & $\begin{array}{l}\text { Numerical } \\
\text { value }\end{array}$ \\
\hline $\begin{array}{l}\text { Ratio of the length of the stator core to its outer } \\
\text { diameter } l_{\delta} / D_{j}\end{array}$ & $2.6 \ldots 3$ \\
\hline $\begin{array}{l}\text { Coefficient of pole overlap } \\
\alpha=b_{p} / \tau\end{array}$ & $0.68 \ldots 0.75$ \\
\hline $\begin{array}{l}\text { Magnet shape coefficient } \\
k_{p}=b_{p} / h_{p}\end{array}$ & $2.1 \ldots 2.5$ \\
\hline $\begin{array}{l}\text { Magnet height coefficient } \\
k_{R}=h_{p} / R_{2}\end{array}$ & $0.1 \ldots 0.16$ \\
\hline $\begin{array}{l}\text { Coefficient of relative air gap value } \\
k_{\delta}=\delta / \tau\end{array}$ & $0.04 \ldots 0.05$ \\
\hline
\end{tabular}

The value of the EMF of the SGPM, and hence the efficiency of the SCEMO, largely depends on the rotational speed of the generator rotor, which is determined by the traffic conditions of the vehicle. Therefore, the energy intensity and parameters of battery, the time of its charge, etc., require special agreement with the parameters of SGPM taking into account the likely characteristics of the movement of a specific type of vehicle.

\section{Conclusions.}

1. An additional power source on board of a vehicle may be a system of electromechanical recuperation of the energy of mechanical oscillations of the vehicle's chassis during its movement into the electric energy of the battery. The analysis confirms the presence of a sufficient amount of energy, which is usually lost in shock absorbers of the vehicle and which can be partially accumulated in battery.

2. The parameters of an important element of the mentioned system - a synchronous generator with permanent magnets, which, due to the peculiarities of its operation, are substantially different from analogous generators of traditional execution, are investigated. The optimal ranges of parameters of SGPM are determined. Their numerical values are presented in the Table and can be used in the development of SGPM in the specified systems.

\section{REFERENCES}

1. Drive2 - Automobile Journal. Electromagnetic suspension Bose. Available at: https://www.drive2.ru/b/721443/ (accessed 13 September 2016). (Rus).

2. Drive2 - Automobile Journal. GenShock - suspension system performing the function of energy regeneration. Available at: https://www.drive2.com/b/668040/ (accessed 11 May 2016). (Rus).

3. Audi MediaCenter. The innovative shock absorber system from Audi. Available at: https://www.audimediacenter.com/en/press-releases/the-innovative-shockabsorber-system-from-audi-new-technology-saves-fuel-andenhances-comfort-6551 (accessed 20 June 2017).

4. Vaskovskyi Yu.M., Haydenko Yu.A. Research of electromagnetic processes in permanent magnet synchronous motors based on a «electric circuit - magnetic field» mathematical model. Technical Electrodynamics, 2018, no.2, pp. 47-54. (Ukr). doi: 10.15407/techned2018.02.047.

Received 15.06.2018

Yu.M. Vaskovskyi ${ }^{1}$, Doctor of Technical Science, Professor, M.V. Poda ${ }^{1}$, Postgraduate Student,

I.V. Koshikar ${ }^{1}$, Master of Science,

${ }^{1}$ National Technical University of Ukraine «Igor Sikorsky Kyiv Polytechnic Institute»,

37, Prospect Peremohy, Kyiv-56, 03056, Ukraine,

e-mail:vun157@gmail.com,poda.mv@gmail.com

How to cite this article:

Vaskovskyi Yu.M., Poda M.V., Koshikar I.V. Electric generator in the recuperation system of the energy from mechanical oscillations in vehicles. Electrical engineering \& electromechanics, 2018, no.5, pp. 24-28. doi: 10.20998/2074-272X.2018.5.04. 